\title{
SEIBERG-WITTEN EQUATIONS AND PSEUDOHOLOMORPHIC CURVES
}

\section{ARMEN SERGEEV}

Communicated by Martin Schlichenmaier

\begin{abstract}
We consider the Taubes correspondence between solutions of SeibergWitten equations on a compact four-dimensional symplectic manifold and pseudoholomorphic curves. We start from Kähler surfaces, in which case there is a direct correspondence between solutions of Seiberg-Witten equations and holomorphic curves. The general Taubes correspondence for symplectic four-manifolds involves, in contrast with the Kähler case, a limiting procedure, called the scaling limit. Under this scaling limit solutions of Seiberg-Witten equations reduce to families of solutions of certain vortex equations in the normal bundle of the limiting pseudoholomorphic curve.
\end{abstract}

\section{Introduction}

One of the most remarkable results, related to the Seiberg-Witten equations (proposed by N. Seiberg and E. Witten in 1994), is the so called "Taubes equation"

$$
\mathrm{SW}=\mathrm{Gr} \text {. }
$$

It is a mnemonic formula, encoding a simple relation between two important invariants of a compact symplectic four-manifold, namely, its Seiberg-Witten invariant, produced from the moduli space of solutions of Seiberg-Witten equations, and the Gromov invariant of this manifold, counting the number of pseudoholomorphic curves in a given homology class.

This "equation" is based on a remarkable construction, proposed by C. Taubes in [9], which associates pseudoholomorphic curves with solutions of SeibergWitten equations. In the first part of the paper we explain how the Taubes correspondence is established in the simpler case of compact Kähler surfaces. In the Kähler case the moduli space of solutions of (perturbed) Seiberg-Witten equations can be identified with the space of effective divisors (i.e., holomorphic curves with multiplicities). This is analogous to the Bradlow's description of the moduli space 
of solutions of vortex equations on compact Riemann surfaces (cf. [2]). In the general case of compact symplectic four-manifolds pseudoholomorphic curves arise in the scaling limit of Seiberg-Witten equations. Solutions of Seiberg-Witten equations under this limit reduce to pseudoholomorphic families of solutions of vortex equations in the normal planes of the limiting pseudoholomorphic curves.

\section{Seiberg-Witten Equations on Kähler Surfaces}

\subsection{Seiberg-Witten Equations}

Let $X$ be a (simply connected) compact Kähler surface, provided with a symplectic two-form $\omega$. Denote by $J$ a complex structure on $X$ which is compatible with $\omega$ in the sense that

- $\omega(J \xi, J \eta)=\omega(\xi, \eta)$ for any tangent vectors $\xi, \eta \in T X$

- the symmetric tensor, defined by

$$
g(\xi, \eta):=\omega(\xi, J \eta)
$$

generates a Riemannian metric on $X$.

Let $E \rightarrow X$ be a complex Hermitian line bundle on $X$, provided with a Hermitian connection $B$.

These data define the following Seiberg-Witten equations on $X$

$$
\left\{\begin{array}{l}
\bar{\partial}_{B} \varphi_{0}+\bar{\partial}_{B}^{*} \varphi_{2}=0 \\
F_{B}^{0,2}+\eta^{0,2}=\frac{\bar{\varphi}_{0} \varphi_{2}}{2} \\
F_{\text {can }}^{\omega}+F_{B}^{\omega}+\eta^{\omega}=\frac{i}{4}\left(\left|\varphi_{0}\right|^{2}-\left|\varphi_{2}\right|^{2}\right) .
\end{array}\right.
$$

In these equations

- $\partial_{B}$ is the covariant exterior derivative, associated with the connection $B$, and $\bar{\partial}_{B}$ is its $(0,1)$-component with respect to $J ; \bar{\partial}_{B}^{*}$ denotes the $L^{2}$-adjoint operator of $\bar{\partial}_{B}$

- $F_{B}$ is the curvature of the connection $B$; accordingly, $F_{B}^{0,2}$ denotes its $(0,2)$-component with respect to $J$ and $F_{B}^{\omega}$ is the component of $F_{B}$, parallel to $\omega$ (i.e., $F_{B}^{\omega}$ coincides with the inner product of $F_{B}$ with $\omega$ ) 
- $2 A_{\text {can }}$ is the canonical connection, generated by the Riemannian metric of $X$, on the anticanonical bundle $K^{*}=\Lambda^{0,2}$ on $X$ (note that in the case when $X$ has a spin structure, i.e., $K^{*}$ admits the square root $L_{\text {can }} \rightarrow X$ such that $K^{*}=L_{\text {can }} \otimes L_{\text {can }}, A_{\text {can }}$ would be a connection on $\left.L_{\text {can }}\right) ; 2 F_{\text {can }}$ is the curvature of the canonical connection $2 A_{\text {can }}$

- $\eta$ is a fixed arbitrary self-dual two-form on $X$ with imaginary coefficients to be considered as a perturbation.

We are looking for solutions of these SW-equations, given by the Hermitian connection $B$ and a spinor field $\Phi=\left(\varphi_{0}, \varphi_{2}\right)$, represented by a pair of sections $\varphi_{0}$, $\varphi_{2}$ where

- $\varphi_{0}$ is a section of the line bundle $E \rightarrow X$, i.e., $\varphi_{0} \in \Omega^{0}(X, E)$

- $\varphi_{2}$ is a $(0,2)$-form on $X$ with values in $E \rightarrow X$, i.e., $\varphi_{2} \in \Omega^{0,2}(X, E)$.

We have written here the Seiberg-Witten equations in the form, adapted to the Kähler case. The standard Seiberg-Witten equations on Riemannian four-manifolds have the following form (cf. [5-7])

$$
\left\{\begin{array}{l}
D_{B} \Phi=0 \\
F_{B}^{+}+\eta=\Phi \otimes \Phi^{*}-\frac{1}{2}|\Phi|^{2} \cdot \mathrm{id} .
\end{array}\right.
$$

The relation between these standard Seiberg-Witten equations and the SW-equations, written above, is established as follows.

The first of RSW-equations, which corresponds to the first of the SW-equations, is the Dirac equation with

$$
D_{B}: \Omega^{0}(X, E) \oplus \Omega^{0,2}(X, E) \longrightarrow \Omega^{0,1}(X, E)
$$

being the covariant Dirac operator, generated by the connection $B$. We can write it in a more familiar way, namely as a homomorphism

$$
D_{B}: \Gamma\left(X, W^{+}\right) \longrightarrow \Gamma\left(X, W^{-}\right)
$$

of sections of the semispinor vector bundles (of rank 2), defined by

$$
W_{E}^{+}:=\Lambda^{0}(X, E) \oplus \Lambda^{0,2}(X, E), \quad W_{E}^{-}:=\Lambda^{0,1}(X, E)
$$

where $\Lambda^{p, q}(X, E)$ denotes the bundle of $(p, q)$-forms on $X$ with values in the line bundle $E$. 
In the second RSW-equation $F_{B}^{+}$is the self-dual part of the curvature $F_{B}$, while in the right hand side we have a traceless Hermitian endomorphism of the semispinor bundle $W_{E}^{+}$, defined by a spinor field $\Phi \in \Gamma\left(X, W^{+}\right)$. To equalize the two sides of the second equation, one should represent the two-form $F_{B}^{+}$with imaginary coefficients as an endomorphism of $\Gamma\left(X, W^{+}\right)$, using the Clifford multiplication (we refer to the above references [5-7] for details). To relate the second RSWequation with the second and third SW-equations, we recall that the bundle of self-dual two-forms on a Kähler surface with pure imaginary coefficients has the following Hodge decomposition

$$
\Lambda_{+}^{2} \otimes \mathrm{i} \mathbb{R}=\left(\Lambda^{2,0} \otimes \mathrm{i} \mathbb{R}\right) \oplus \mathrm{i} \mathbb{R}[\omega] \oplus\left(\Lambda^{0,2} \otimes \mathrm{i} \mathbb{R}\right)
$$

where the first and the third terms on the right are complex conjugate to each other. So we can split the second RSW-equation into three components, according to the above decomposition of $\Lambda_{+}^{2} \otimes i \mathbb{R}$. The second and third of SW-equations are precisely the $(0,2)$ and $(1,1)$ components of the second RSW-equation.

We return to the Seiberg-Witten SW-equations on the Kähler surface $X$. These equations are invariant under gauge transformations, given by the natural action

$$
B \longmapsto B+u^{-1} d u, \quad \Phi \longmapsto u^{-1} \Phi
$$

of the group $\mathcal{G}:=\Gamma(X, \mathrm{U}(1))$ of gauge transformations. We are interested in the moduli space of SW-solutions, i.e., the space of solutions of SW-equations modulo gauge transformations.

\subsection{Solvability Conditions}

We analyze first the solvability conditions for the SW-equations, assuming that the perturbation two-form $\eta$ is of the $(1,1)$-type.

By applying the $\bar{\partial}_{B}$-operator to the first SW-equation, we get

$$
\bar{\partial}_{B} \bar{\partial}_{B}^{*} \varphi_{2}=-\bar{\partial}_{B} \bar{\partial}_{B} \varphi_{0}=-F_{B}^{0,2} \varphi_{0}=-\frac{\left|\varphi_{0}\right|^{2} \varphi_{2}}{2}
$$

where in the last equality we have used the second SW-equation. Integrating this relation over $X$ with $\bar{\varphi}_{2}$, we obtain

$$
\left\|\bar{\partial}_{B}^{*} \varphi_{2}\right\|_{L^{2}}^{2}+\frac{1}{2}\left\|\varphi_{0}\right\|_{L^{2}}^{2} \cdot\left\|\varphi_{2}\right\|_{L^{2}}^{2}=0
$$

It follows that

$$
\bar{\partial}_{B}^{*} \varphi_{2}=\bar{\partial}_{B} \varphi_{0}=\bar{\varphi}_{0} \varphi_{2} \equiv 0
$$


It is easy to prove that solutions of $\bar{\partial}_{B}$ (and $\bar{\partial}_{B}^{*}$ ) equation satisfy to the following identity principle: if such a solution vanishes on an open subset of $X$, it is identically zero on $X$. By this principle the last equation implies that either $\varphi_{0}$ or $\varphi_{2}$ should be identically zero on $X$.

In order to detect which of them is identically zero, we integrate the third SWequation. Then we get

$$
\begin{aligned}
\frac{\left\|\varphi_{0}\right\|_{L^{2}}^{2}-\left\|\varphi_{2}\right\|_{L^{2}}^{2}}{2} & =\int_{X} \frac{\left|\varphi_{0}\right|^{2}-\left|\varphi_{2}\right|^{2}}{4} \omega \wedge \omega=\mathrm{i} \int_{X}\left(F_{\text {can }}+F_{B}+\eta\right) \wedge \omega \\
& =\pi\left(-c_{1}(K)+2 c_{1}(E)\right) \cdot[\omega]+\mathrm{i} \int_{X} \eta \wedge \omega .
\end{aligned}
$$

Consider first the case when $\eta=0$, corresponding to the non-perturbed SeibergWitten equations. Then the above relation (2) reduces to

$$
\left\|\varphi_{2}\right\|^{2}-\left\|\varphi_{0}\right\|^{2}=2 \pi\left(2 c_{1}(E) \cdot[\omega]-c_{1}(K) \cdot[\omega]\right) .
$$

This relation implies the following solvability conditions

- if $c_{1}(E) \cdot[\omega]>c_{1}(K) \cdot[\omega] / 2$, then $\varphi_{0} \equiv 0, \varphi_{2} \not \equiv 0$

- if $c_{1}(E) \cdot[\omega]<c_{1}(K) \cdot[\omega] / 2$, then $\varphi_{0} \not \equiv 0, \varphi_{2} \equiv 0$.

We provide now our line bundle $E$ with a holomorphic structure, determined by the $\bar{\partial}_{B}$-operator. Recall the following general fact: if the holomorphic line bundle $\left(E, \bar{\partial}_{B}\right)$ has a non-trivial holomorphic section $\varphi_{0}$, then

$$
c_{1}(E) \cdot[\omega] \geq 0 .
$$

We can apply the same argument to the holomorphic line bundle $K \otimes E^{*}$, provided with the holomorphic structure, generated by the $\bar{\partial}_{B}^{*}$-operator. If this bundle has a non-trivial holomorphic section $\varphi_{2}$, then

$$
c_{1}(K) \cdot[\omega] \geq c_{1}(E) \cdot[\omega] .
$$

Thus we have the following solvability conditions in terms of the Chern class

- if $0<c_{1}(E) \cdot[\omega]<c_{1}(K) \cdot[\omega] / 2$, then $\varphi_{0}$ is a non-trivial solution of SW-equations and $\varphi_{2} \equiv 0$

- if $c_{1}(K) \cdot[\omega] / 2<c_{1}(E) \cdot[\omega]<c_{1}(K) \cdot[\omega]$, then $\varphi_{2}$ is a non-trivial solution of SW-equations and $\varphi_{0} \equiv 0$. 
In the next section, where we analyze the existence of solutions of the general (perturbed) SW-equations, we shall obtain the solvability conditions for the perturbed equations, analogous to the above conditions in the non-perturbed case.

\subsection{Solution of the Seiberg-Witten Equations}

We consider now the perturbed SW-equations in the case of the trivial bundle $E$ and choose the perturbation $\eta$ in the form

$$
\eta=-F_{\text {can }}^{+}+\mathrm{i} \lambda \omega
$$

where $\lambda>0$ is a parameter. As in the previous section, we integrate the third SW-equation. We arrive at

$$
4 \lambda \cdot \operatorname{Vol}(X)+\left\|\varphi_{2}\right\|^{2}-\left\|\varphi_{0}\right\|^{2}=0 .
$$

It follows that $\varphi_{2} \equiv 0$ and the $\mathrm{SW}$-equations take on the form

$$
\bar{\partial}_{B} \varphi_{0}=0, \quad F_{B}^{0,2}=0, \quad 4 \mathrm{i} F_{B}^{\omega}=4 \lambda-\left|\varphi_{0}\right|^{2} .
$$

Since $E$ is trivial, these equations admit the trivial solution

$$
B \equiv 0, \quad \varphi_{2} \equiv 0, \quad \varphi_{0} \equiv 2 \sqrt{\lambda} .
$$

It may be proved (cf. $[6,7]$ ) that this solution is unique (up to gauge transformations).

We switch now to the case of a general $E$ and choose the perturbation $\eta$ in the form

$$
\eta=\mathrm{i} \lambda \omega
$$

where $\lambda>0$ is a parameter, as above. We integrate again the third SW-equation in order to deduce the following necessary solvability condition

$$
0 \leq c_{1}(E) \cdot[\omega]<c_{1}(K) \cdot[\omega] / 2+\lambda \operatorname{Vol}(X) .
$$

It turns out that this condition is also sufficient for the solvability of SW-equations. This assertion is analogous to the Bradlow's theorem on the solvability of vortex equations on compact Riemann surfaces (cf. [2]) and is proved in a similar way. We refer to [2] (or to [7]) for a detailed proof and give here only the idea of Bradlow's construction.

It's easy to show, as above, that any solution of SW-equations under the condition (3) is represented by a Hermitian connection $B$ on the line bundle $E$ and a spinor field $\varphi_{0}$ (i.e., $\varphi_{2}$ vanishes identically under the condition (3)). The moduli space of SW-solutions $\left(B, \varphi_{0}\right)$ under the condition (3) admits the following description. 
Proposition 1. Under the condition (3) there is a one-to-one correspondence between the moduli space

$$
\frac{\left\{\mathrm{SW} \text {-solutions }\left(B, \varphi_{0}\right)\right\}}{\mathcal{G}}
$$

of Seiberg-Witten solutions and the space of effective divisors of degree $c_{1}(E)$ on $X$.

This Proposition is proved in the following way. It is well known that the space of effective divisors can be identified with the space

\{holomorphic line bundles with a non-trivial holomorphic section\}

$$
\text { \{holomorphic equivalence\} }
$$

of holomorphic equivalence classes of holomorphic line bundles $\left(E, \bar{\partial}_{E}\right)$ over $X$ with a non-trivial holomorphic section $\varphi_{0}$.

Since $\bar{\partial}_{E}=\bar{\partial}_{B}$ for an appropriate Hermitian connection $B$, the space of holomorphic line bundles with non-trivial holomorphic sections can be, in its turn, identified with the space of solutions $\left(B, \varphi_{0}\right)$ of the equations

$$
\bar{\partial}_{B} \varphi_{0}=0, \quad F_{B}^{0,2}=0
$$

modulo complex gauge transformations from the complexified group $\mathcal{G}_{\mathbb{C}}$.

To prove that the latter space can be identified with the original Seiberg-Witten moduli space, we must show that for any solution $\left(B, \varphi_{0}\right)$ of the equations (4)

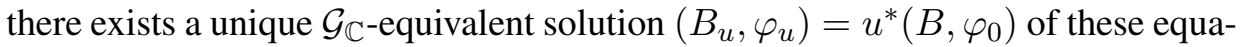
tions, which satisfies the third SW-equation. Writing down the gauge factor $u$ in the form $u=\mathrm{e}^{\theta}$ for a real-valued function $\theta \in \mathbb{R}$, we obtain the following Liouville-type equation for $\theta$

$$
8 \mathrm{i}(\partial \bar{\partial} \theta)^{\omega}+\mathrm{e}^{-2 \theta}\left|\varphi_{0}\right|^{2}=4 \pi \lambda-4 \mathrm{i}\left(F_{B}^{\omega}+F_{\text {can }}^{\omega}\right) .
$$

According to the Kazdan-Warner theorem [4], this equation has a unique solution under the condition (3).

We can reformulate the above Proposition in the following way: for sufficiently large $\lambda$ (such that (3) is satisfied) the moduli space of SW-solutions can be identified with the space of complex curves on $X$ with multiplicity $c_{1}(E)$. In the second part of this paper we'll see that in the symplectic case the moduli space of SWsolutions can be identified with the space of pseudoholomorphic divisors on $X$ of multiplicity $c_{1}(E)$. However, in contrast with the Kähler case, this identification will require the passing to the limit $\lambda \rightarrow \infty$. 


\section{Seiberg-Witten Equations on Symplectic Four-Manifolds}

\subsection{Seiberg-Witten Equations and Solvability Conditions}

Let $(X, \omega)$ be a (simply connected) compact symplectic four-manifold, provided with a symplectic two-form $\omega$. We denote by $J$ an almost complex structure on $X$, compatible with $\omega$ in the sense of Section 1.1. We fix again a complex Hermitian line bundle $E \rightarrow X$, provided with a Hermitian connection $B$, and choose the perturbation two-form

$$
\eta=-F_{\text {can }}^{+}+\frac{i \lambda}{4} \omega .
$$

Since we are going to take the limit $\lambda \rightarrow \infty$, it is convenient to introduce the normalized sections $\alpha \in \Omega^{0}(X, E), \beta \in \Omega^{0,2}(X, E)$ by setting

$$
\alpha:=\frac{\varphi_{0}}{\sqrt{\lambda}}, \quad \beta:=\frac{\varphi_{2}}{\sqrt{\lambda}} .
$$

The SW-equations for these sections will have the form

$$
\left\{\begin{array}{l}
\bar{\partial}_{B} \alpha+\bar{\partial}_{B}^{*} \beta=0 \\
\frac{2}{\lambda} F_{B}^{0,2}=\bar{\alpha} \beta \\
\frac{4 \mathrm{i}}{\lambda} F_{B}^{\omega}=1+|\beta|^{2}-|\alpha|^{2}
\end{array}\right.
$$

similar to the SW-equations in the Kähler case.

However, for the analysis of the solvability of these equations we cannot use the same idea, as in the Kähler case. The reason is that the application of the $\bar{\partial}_{B^{-}}$ operator to the first $\mathrm{SW}_{\lambda}$-equation yields, instead of the identity (1) in Section 1, the following relation

$$
\bar{\partial}_{B} \bar{\partial}_{B}^{*} \beta=-\bar{\partial}_{B} \bar{\partial}_{B} \alpha=-F_{B}^{0,2} \alpha+\frac{1}{4}\left(\partial_{B} \alpha\right) \circ N_{J} .
$$

The right hand side of (5) contains, compared to (1), an extra term, depending on the Nijenhuis tensor $N_{J}$ of the almost complex structure $J$. This tensor measures the non-integrability of $J$ (we refer, e.g., to [1] for its definition). Since we have no control of $N_{J}$, we get, instead of the integral identity in Section 2.1, only the following inequality, necessary for the solvability of $\mathrm{SW}_{\lambda}$-equations (cf. [6,9])

$$
0 \leq c_{1}(E) \cdot[\omega] \leq c_{1}(K) \cdot[\omega] .
$$


Note that the bounds of the solvability domain (in terms of $c_{1}(E) \cdot[\omega]$ ) remain the same, as in the Kähler case, but in the symplectic case the $\mathrm{SW}_{\lambda}$-equations do not split in general. As in the Kähler case, the equality in the left " $\leq$ "-sign is attained only for the trivial bundle $E$, while the equality in the right " $\leq$ "-sign is attained only when $E$ coincides with the canonical bundle $K$.

\subsection{Associating a Pseudoholomorphic Curve with a Seiberg-Witten Solution}

Solutions of $\mathrm{SW}_{\lambda}$-equations, given by $\left(B_{\lambda}, \Phi_{\lambda}\right)$ with $\Phi_{\lambda}:=\left(\alpha_{\lambda}, \beta_{\lambda}\right)$, satisfy the following a priori estimates, proved in [9]

$$
\left\{\begin{array}{l}
\left|\alpha_{\lambda}\right| \leq 1+\frac{C_{1}}{\lambda} \quad\left|\beta_{\lambda}\right|^{2} \leq \frac{C_{2}}{\lambda}\left(1-|\alpha|^{2}\right)+\frac{C_{3}}{\lambda^{3}} \\
\left\|\bar{\partial}_{B_{\lambda}} \alpha_{\lambda}\right\|^{2}+\left\|\mathrm{d}_{B_{\lambda}} \beta_{\lambda}\right\|^{2} \leq \frac{C_{4}}{\lambda} \\
\left|F_{B_{\lambda}}^{ \pm}\right| \leq C_{5} \lambda\left(1-\left|\alpha_{\lambda}\right|^{2}\right)+C_{6} \\
2 \pi c_{1}(E) \cdot[\omega]-\frac{C_{7}}{\lambda} \leq\left.\frac{\lambda}{2} \int_{X}|1-| \alpha_{\lambda}\right|^{2} \mid \mathrm{d} \text { vol } \leq 2 \pi c_{1}(E) \cdot[\omega]+\frac{C_{7}}{\lambda}
\end{array}\right.
$$

where $C_{1}, \cdots, C_{7}$ are some constants, depending only on $c_{1}(E)$ and almost complex structure $J$.

These estimates imply that the $\mathrm{SW}_{\lambda}$-solution $\left(B_{\lambda}, \Phi_{\lambda}\right)$ have the following behavior for $\lambda \rightarrow \infty$

- $\left|\alpha_{\lambda}\right| \rightarrow 1$ almost everywhere on $X$ (more precisely, outside its zeros)

- $\left\|\bar{\partial}_{B_{\lambda}} \alpha_{\lambda}\right\| \rightarrow 0$, i.e., $\alpha_{\lambda}$ tends to become a $\bar{\partial}_{B_{\lambda}}$-holomorphic section of $E$

- $\beta_{\lambda} \rightarrow 0$ everywhere (together with its first derivatives).

Hence, the situation for $\lambda \rightarrow \infty$ becomes more and more similar to the Kähler one.

Since $\left|\alpha_{\lambda}\right| \rightarrow 1$ everywhere outsides its zeros, it's natural to ask what happens to the zeros of $\alpha_{\lambda}$. Let us denote by

$$
C_{\lambda}:=\alpha_{\lambda}^{-1}(0)
$$

the zero-divisor of $\alpha_{\lambda}$. The above estimates imply (cf. [9]) that the curves $C_{\lambda}$ have a weak limit (in the sense of currents) and this limit is a pseudoholomorphic divisor $C$, assigned to the $\left(\mathrm{SW}_{\lambda}\right)$-solution $\left(B_{\lambda}, \Phi_{\lambda}\right)$. 
In more detail, we associate with a $\mathrm{SW}_{\lambda}$-solution $\left(B_{\lambda}, \Phi_{\lambda}\right)$ the integration current:

$$
F_{\lambda}(\sigma):=\frac{\mathrm{i}}{2 \pi} \int_{X} F_{B_{\lambda}} \wedge \sigma
$$

for a smooth two-form $\sigma \in \Omega^{2}(X, \mathbb{R})$. The norms of the currents $F_{\lambda}$, equal to

$$
\left\|F_{\lambda}\right\|=\sup _{0 \neq \sigma \in \Omega^{2}} \frac{\left|F_{\lambda}(\sigma)\right|}{\sup _{x \in X}|\sigma(x)|}
$$

are uniformly bounded, since the third and fourth of the estimates (6) imply that

$$
\left\|F_{\lambda}\right\| \leq \frac{1}{2 \pi}\left\|F_{B_{\lambda}}\right\|_{L^{1}}<C
$$

where $C>0$ is a constant, not depending on $\lambda$. Hence, we can find a sequence $\lambda_{n} \rightarrow \infty$ such that $F_{\lambda_{n}}$ converges weakly to some $\mathcal{F}$, which is a closed positive integral $(1,1)$-current, Poincaré dual to $c_{1}(E)$. The support of this current $\mathcal{F}$ is the desired pseudoholomorphic curve $C$.

As it is shown in [9], this $C$ is a pseudoholomorphic divisor of degree $d=$ $c_{1}(E)$. It means, in other words, that

$$
C=\sum_{j=1}^{k} d_{j} C_{j}, \quad d_{j} \text { are positive integers }
$$

is the sum of non-intersecting (maybe, non smooth) pseudoholomorphic curves $C_{j}$ with multiplicities $d_{j}$ such that the homology class $[C]$ of the divisor $C$ is equal to

$$
[C]:=\sum d_{j}\left[C_{j}\right]=P D\left(c_{1}(E)\right) .
$$

\subsection{The Limiting Behavior of Seiberg-Witten Solutions}

We analyze next the limiting behavior of a $\mathrm{SW}_{\lambda}$-solution $\left(B_{\lambda}, \Phi_{\lambda}\right)$ for $\lambda \rightarrow \infty$. This limit can be considered as the adiabatic (scaling) limit of $\mathrm{SW}_{\lambda}$-equations (cf. [8]). Let us assume for the simplicity of formulations that the limiting divisor $C$ is smooth and $k=1$ so that $[C]=d\left[C_{0}\right]$ where $C_{0}$ is a smooth pseudoholomorphic curve.

Then it can be shown that for a suitable choice of a subsequence $\lambda_{n} \rightarrow \infty$ and representatives in the gauge classes $\left[B_{\lambda_{n}}, \Phi_{\lambda_{n}}\right]$ the subsequence $\left(B_{n}, \Phi_{n}\right) \equiv$ 
$\left(B_{\lambda_{n}}, \Phi_{\lambda_{n}}\right)$ converges for $n \rightarrow \infty$ to a family $(A, \alpha)$ of solutions of vortex equations on the normal planes $N_{z}$, parametrized by $z \in C_{0}$, where $N \rightarrow C_{0}$ is the normal bundle of $C_{0}$. The zero-divisor of $\alpha$ is equal to $C$.

Recall that the vortex equations on the complex plane $\mathbb{C}$ have the form (cf. [3])

$$
\left\{\begin{array}{l}
\bar{\partial}_{A} \alpha=0 \\
F_{A}=*\left(1-|\alpha|^{2}\right) .
\end{array}\right.
$$

A solution of these equations is given by a Hermitian connection $A$ on $\mathbb{C}$ and a complex-valued function $\alpha$ on $\mathbb{C}$ such that the energy of $(A, \alpha)$, given by

$$
\int\left\{\left|F_{A}\right|^{2}+\left|\mathrm{d}_{A} \alpha\right|^{2}+\frac{1}{4}\left(1-|\alpha|^{2}\right)^{2}\right\} \mathrm{d} \text { vol }
$$

is finite. Solutions of vortex equations minimize the energy (7) in a given topological class, determined by the algebraic number $d$ of zeros of $\alpha$ (called, otherwise, the vortex number). The above equations are invariant under gauge transformations of the form

$$
A \longmapsto A+\mathrm{i} d \chi, \quad \alpha \longmapsto \mathrm{e}^{-\mathrm{i} \chi} \alpha
$$

where $\chi$ is a real-valued function on $\mathbb{C}$. We denote by $\mathcal{M}_{d}$ the moduli space of solutions $(A, \alpha)$ of the above vortex equations with vortex number $d>0$ modulo gauge transformations. It is well known (cf. [3]) that this moduli space may be identified with the $d$ th symmetric power of $\mathbb{C}$

$$
\mathcal{M}_{d} \cong \operatorname{Sym}^{d} \mathbb{C} \cong \mathbb{C}^{d} .
$$

We return after this short digression to the Seiberg-Witten equations. As we have remarked, the limit of $\mathrm{SW}_{\lambda_{n}}$-solutions $\left(B_{n}, \Phi_{n}\right)$ for $n \rightarrow \infty$ is a family $\left(A_{z}, \alpha_{z}\right)$ of solutions of vortex equations on the normal planes $N_{z} \approx \mathbb{C}$, parametrized by $z \in C_{0}$. We can consider the limiting family $(A, \alpha)$ as a complex path

$$
\gamma: z \in C_{0} \longmapsto\left[A_{z}, \alpha_{z}\right] \in \mathcal{M}_{d}
$$

in the moduli space $\mathcal{M}_{d}$ where $\left[A_{z}, \alpha_{z}\right]$ denotes the gauge class of $\left(A_{z}, \alpha_{z}\right)$. This path is not arbitrary - it should be pseudoconvex with respect to a suitable almost complex structure on $\mathcal{M}_{d}$ (this condition corresponds, in a general context of the adiabatic limit, to the adiabatic equation, cf. [8]).

In the reverse direction it can be shown (cf. [10]) that for any pseudoholomorphic path $\gamma$ in $\mathcal{M}_{d}$ and, moreover, for any pseudoholomorphic section $\gamma$ of the vortex bundle

$$
N \otimes_{\mathrm{U}(1)} \mathcal{M}_{d} \longrightarrow C_{0}
$$

over a pseudoholomorphic curve $C_{0}$, sufficiently close to the zero section of $N \rightarrow$ $C_{0}$, there exists a $\mathrm{SW}_{\lambda}$-solution $\left(B_{\lambda}, \Phi_{\lambda}\right)$, which approximates $\gamma$ for $\lambda \rightarrow \infty$. 


\section{Acknoledgements}

The author was partly supported by the Russian Foundation for Basic Research (grant 04-01-00236), the Program for Support of Scientific Schools (grant NSh1542.2003.1) and the Scientific Program "Nonlinear Dynamics" of RAS.

\section{References}

[1] Besse A., Einstein Manifolds, Springer, Berlin, 1987.

[2] Bradlow S., Vortices in Holomorphic Line Bundles over Closed Kähler Manifolds, Comm. Math. Phys. 135 (1990) 1-17.

[3] Jaffe A. and Taubes C., Vortices and Monopoles, Birkhäuser, Boston, 1980.

[4] Kazdan J. and Warner F., Curvature Functions for Compact 2-Manifolds, Ann. Math. 99 (1974) 14-47.

[5] Moore J., Lectures on Seiberg-Witten Invariants, Lecture Notes Math. 1629, Springer, Berlin, 1996.

[6] Salamon D., Spin Geometry and Seiberg-Witten Invariants, Warwick Univ. preprint, Warwick, 1996.

[7] Sergeev A., Vortices and Seiberg-Witten Equations, Nagoya Math. Lectures vol. 5, Nagoya Univ., Nagoya, 2002.

[8] Sergeev A., Adiabatic Limit in the Seiberg-Witten Equations, Amer. Math. Soc. Transl. 212 (2004) 281-295.

[9] Taubes C., $S W \Rightarrow G r$ : From the Seiberg-Witten Equations to Pseudoholomorphic Curves, J. Amer. Math. Soc. 9 (1996) 845-918.

[10] Taubes C., Gr $\Rightarrow$ SW: From Pseudo-holomorphic Curves to Seiberg-Witten Solutions, J. Diff. Geom. 51 (1999) 203-334.

Armen Sergeev

Steklov Mathematical Institute

Gubkina 8, 119991, Moscow

RUSSIA

E-mail address: sergeev@mi.ras.ru 